\title{
GESTÃO DE ESTOQUE DE ITENS DE CONSUMO EM UMA EMPRESA ALIMENTÍCIA.
}

\author{
M.E.M.S.Celestino ${ }^{1, *}$, C.E.Bastos ${ }^{1}$ \\ ${ }^{1}$ Faculdade de Tecnologia de São José dos Campos - Professor Jessen Vidal \\ Av. Cesare Mansueto Giulio Lattes, 1350 - Eugênio de Melo, São José dos Campos/SP, \\ CEP.: 12247-014, Brasil. \\ Telefone: (12) 3905-2423 \\ "maeduardamsc@gmail.com
}

\begin{abstract}
RESUMO: Ter uma boa gestão de estoques colabora para que a empresa se mantenha competitiva através da redução de custos com armazenamento de materiais desnecessários. $\mathrm{O}$ presente artigo tem como objetivo analisar os estoques de forma a manter níveis adequados de material e atender as demandas diárias, de modo que a produção não seja afetada por sua falta. Após uma revisão bibliográfica associada à coleta de dados de estoque em uma empresa do setor alimentício, foi possível modelar um processo de gestão baseado na aplicação de ferramentas de gestão de estoques, como estoque de segurança e curva ABC. Os resultados apontam que os métodos utilizados são viáveis e auxilia os gestores na tarefa de definir os estoques de segurança que melhor se aplicam a sua realidade. Garantir uma boa qualidade de seus produtos e que seus clientes estejam satisfeitos é uma das missões da empresa.
\end{abstract}

PALAVRAS-CHAVE: Gestão de Estoque; Estoque de Segurança; Classificação ABC; Coleta de Dados; Nível de Serviço.

ABSTRACT: Having a good inventory management helps the company stay competitive by reducing the cost of storing unnecessary materials. The objective of this article is to analyze the inventories in order to maintain adequate levels of material and meet the daily demands, so that the production is not affected by its lack. After a bibliographic review associated with the collection of inventory data in a food company, it was possible to model a management process based on the application of inventory management tools, such as safety stock and ABC curve. The results indicate that the methods used are feasible and assists managers in the task of defining the security stocks that best apply to their reality. New jobs can broaden the range of sectors and products surveyed as well as use other methods of analysis in order to mature the proposed process.

KEYWORDS: Inventory Management; Safety stock; ABC classification; Data collect; Service level.

\section{INTRODUÇÃO.}

Pode-se considerar que um aspecto relevante para se estabilizar a posição de uma empresa produtiva é a estratégia da gestão de estoques. Ballou (2006) considera o estoque um ativo de alto custo para as empresas e manter materiais que não são necessários acarreta em desaproveitamento do espaço físico e também de investimentos.

Segundo Wanke (2011), a falta de ferramentas adequadas para a gestão de estoque tem muitos efeitos negativos principalmente no que se refere aos custos desnecessários com materiais com pouca ou nenhuma movimentação dentro de um armazém. Machado e Espinha (2005) 
constataram na época de seus estudos uma carência das empresas brasileiras no que diz respeito às tecnologias que apoiam esses processos logísticos. Desde então, é notável que a tecnologia de informação passou a ser uma forte aliada da logística, principalmente no que se refere à logística interna. $\mathrm{O}$ uso de sistemas tipo ERPs (Enterprise Resource Planning) tem sido amplamente difundido dentro das grandes companhias, fato esse que contribui com a informatização da gestão de estoque e a possibilidade de uma gestão em tempo real.

Apesar de o contexto atual ser favorável, a dificuldade de integração dos sistemas e a especificidade das operações das empresas são fatores ainda não resolvidos no contexto da gestão de estoque. Isso é demonstrado nos trabalhos de Machado e Espinha (2005).

Esse artigo expõe conceitos e aplicações de técnicas de gestão de estoque, tais como, a classificação ABC (Regra de Pareto), Estoque de Segurança e Ponto de Pedido, que podem trazer grandes benefícios para a empresa.

Nesse contexto, a gestão de estoques surge como balizador para agregar valor ao cliente, de modo que as empresas buscam atender o cliente com a quantidade ideal e o mais rápido possível buscando a fidelização (BALLOU, 2006).

Novaes (2015) aponta que a estratégia de estoque é vinculada a outras atividades logísticas, das quais se destacam: armazenagem e transporte. Esse trabalho está focado na conciliação em atender a um processo estratégico e não somente operacional. Chopra e Meindl (2003) indicam que estratégias de layout e simulação podem ser aplicadas para redução de movimentações internas e otimização dos volumes de estoque.

O objetivo geral é analisar os estoques de forma a manter níveis adequados de material aumentando assim a competitividade de uma empresa.

Para a consecução do objetivo geral foram estabelecidos os seguintes objetivos específicos:

- Realizar pesquisas bibliográficas sobre gestão de estoque;

- Avaliar a situação atual do estoque dos itens em estudo;

- Coletar dados de movimentação e compra dos itens;

- Propor melhorias por meio de ferramentas de gestão para gerir de forma eficiente o estoque.

\subsection{Metodologia} técnicos.

A pesquisa foi estruturada em quatro pilares: natureza, abordagem, objetivos e procedimentos

Quanto à natureza, decidiu-se por adotar uma pesquisa aplicada. Assim, serão aplicados conhecimentos práticos para a resolução de problemas específicos.

$\mathrm{Na}$ abordagem, trata-se de uma pesquisa qualitativa e quantitativa.

Os objetivos consistem em exploratórios e descritivos. Gil (2002) aponta que a pesquisa exploratória permite uma maior familiarização com o tema em estudo. A pesquisa descritiva busca trazer análises por meio de estudos de caso e análises documentais.

Por fim, os procedimentos técnicos consistem em pesquisa bibliográfica e pesquisa de levantamento. A pesquisa bibliográfica auxilia na discussão da literatura específica do tema, trazendo conhecimento sobre o que já foi publicado e estudado sobre o tema em estudo (FONSECA, 2002). Quanto à pesquisa de levantamento, esse trabalho tem por base a busca de amostra de dados que permitem a aplicação de métodos matemáticos para se chegar a uma conclusão referente ao tema em estudo, que é a gestão dos itens de consumo em uma empresa alimentícia. 


\section{FUNDAMENTAÇÃO TEÓRICA.}

De acordo com Slack (2009) e Ballou (2006) o estoque se trata de recursos armazenados em um sistema de transformação. Uma acumulação de matérias-primas, suprimentos, componentes, materiais em processos e produto acabados que aparecem em muitos pontos dentro do canal de produção e logística de uma empresa. Alguns tratam o estoque como qualquer recurso transformador de capital. Devido ao desequilibro existente entre fornecimento e demanda, a gestão de estoque acaba se tornando um elemento imprescindível dentro da organização e o meio com que a organização administra e cuida de seus estoques impacta diretamente na lucratividade e competitividade da mesma em relação ao mercado. (BERTAGLIA, 2009).

\subsection{Tipos de Estoques}

Slack (2009), afirma que existem cinco tipos de estoque: estoque de segurança, estoque de ciclo, estoque de desacoplamento, estoque de antecipação e estoque no canal. Nesse artigo, o foco será no estoque de segurança devido ao tipo de material que será tratado.

\subsubsection{Estoque de Segurança}

Slack (2009) também o chama de estoque isolador. Seu principal objetivo é combater as incertezas existentes no fornecimento e na demanda. Trata-se de uma quantidade determinada, através de procedimentos estatísticos, que será acrescentada ao estoque normal necessário para alimentar as condições de demanda e fornecimento médio.

Ballou (2006) destaca a extrema importância de um elevado nível de previsão para que seja possível a redução dos níveis do estoque de segurança e ele ainda completamente que se existisse a possibilidade de prever com absoluta certeza a demanda, não existiria a necessidade de um nível de estoque de segurança.

O estoque de segurança (ES) leva em consideração a demanda e o desvio padrão, considerando a oscilação da demanda durante o tempo de reposição e pode ser calculado a partir da Equação (1), onde $Z \alpha$ representa o coeficiente da tabela de distribuição normal, que indica a possibilidade de haver uma demanda maior que o estoque de segurança, o fator considerado é o nível de serviço que pretende-se manter enquanto o estoque de segurança é consumido; Do indica o desvio padrão da demanda durante o período de atendimento e TA é o tempo de atendimento desse pedido, também conhecido como lead time (SLACK 2009).

$$
\mathrm{ES}=\mathrm{Z} \alpha * \mathrm{D} \sigma * \sqrt{\mathrm{TA}}
$$

\subsection{Ponto de Pedido}

Nesse modelo, o pedido é realizado quando ainda há itens suficientes no estoque para atender a demanda durante o período entre a data em que foi iniciado o processo de compra até que seja finalizado. A Equação 2 é utilizada para calcular o ponto de pedido ideal, onde $\mathrm{PP}=$ Ponto de Pedido, $\mathrm{CM}=$ Consumo Médio, $\mathrm{TR}=$ Tempo de Ressuprimento e ES= Estoque de Segurança, apresenta o momento em que se deve realizar um novo pedido, ou seja, o ponto de pedido.

$$
\mathrm{PP}=(\mathrm{CM} * \mathrm{TR})+\mathrm{ES}
$$

A Figura 1 mostra a representação gráfica do estoque de segurança e ponto de reposição. 
Figura 1. Estoque de segurança e ponto de reposição.

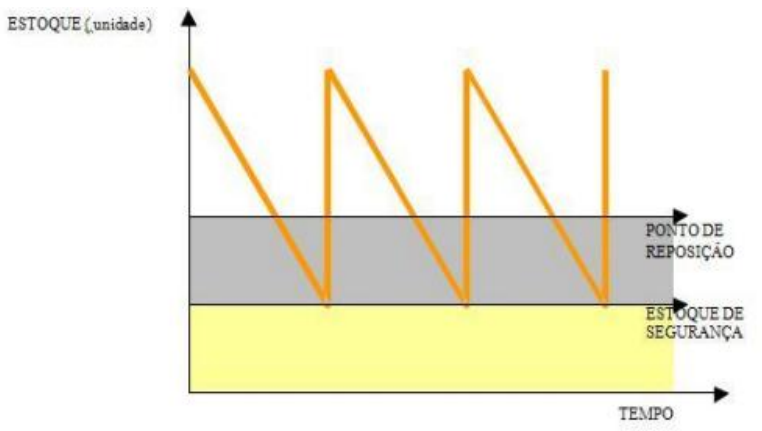

\subsection{Classificação ABC}

Para uma empresa que mantém uma grande variedade de itens em seu catálogo, sempre haverá aqueles que acabam se tornando mais importante do que os outros. Seja pela alta taxa de uso ou pelo alto valor agregado, o que no último caso, uma quantidade de estoque mal projetada poderia gerar custos altíssimos para a empresa.

O controle de estoque através deste método fornece a possibilidade de a empresa focalizar seus esforços nos itens mais importante de seu estoque, seja pelo valor, pelo volume de utilização ou qualquer outro tipo de parâmetro que seja mais adequado (BERTAGLIA, 2009).

Este método tem como meio a divisão dos itens em três categorias:

- Itens de classe A: No topo se encontra os $20 \%$ daqueles itens em que, juntos, representam cerca de $80 \%$ de todo o valor do estoque.

- Itens de classe B: Na faixa do meio estão os de médio valor, cerca de $30 \%$ em que se concentra em média $10 \%$ do valor total do estoque.

- Itens de classe C: Por fim está à faixa mais baixa representada pelos itens de menor valor.

Figura 2. Gráfico Classificação ABC.

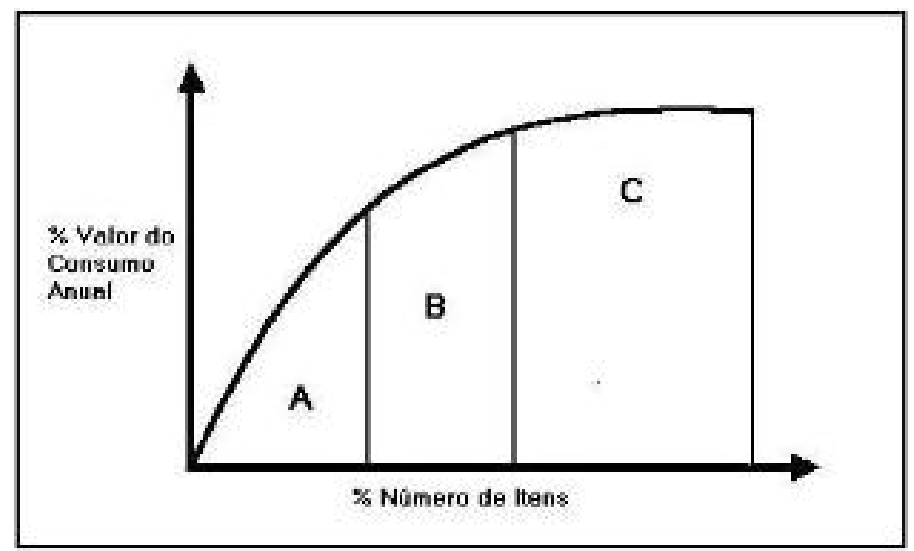

\subsection{Nìvel de Serviço}

Para Ballou (2006), o nível de serviço é um valor de grande relevância que as empresas oferecem a seus clientes a fim de conquistá-los e garantir que retornem em busca de novos serviços. Novaes (2015) ressalta a importância de analisar as particularidades dos processos de abastecimento e entrega, de modo que os materiais sejam entregues nos lugares certos, no momento desejado, 
atendendo o nível de serviço desejado pelo cliente e com o menor custo possível para a realização do processo e garantindo que o cliente não seja prejudicado no momento em que o nível de estoque esteja mais baixo, desse modo, é importante garantir que o ponto de pedido seja ideal para utilização desse material na empresa.

Tendo em mente que se as empresas buscam potencializar a satisfação de seus clientes, muitas vezes correm o risco de aumentar os custos do processo de abastecimento, pois atendem pedidos constantes com pouca carga e em horários flexíveis, gerando mais custos de transporte (KOBAYASHI, 2000)

A disponibilidade, o desempenho e a confiabilidade são apontados como os três elementos indispensáveis para que uma prestadora de serviços garanta a satisfação de seus clientes. A disponibilidade é a eficácia de ter o produto no momento em que o cliente necessitar, isso implica na armazenagem e ainda faz uso do conceito de estoque de segurança. Desempenho é caracterizado pelo cumprimento dos prazos de entrega estipulados ou com uma variação aceitável estipulada pelo cliente. E por fim a confiabilidade une os dois fatores de serviço citados e ainda acrescenta a capacidade de oferecer ao cliente informações sobre as condições de seu pedido para que o mesmo se planeje e execute suas atividades (BOWERSOX E CLOSS 2000).

\section{APLICAÇÃO}

Foi escolhida uma empresa do ramo alimentício, nome fantasia da empresa é MEMSC LTDA, para estudo de estoque de itens de uso e consumo. Esses itens são de extrema importância para o processo produtivo da empresa, pois corroboram com a garantia da qualidade dos produtos e proporcionam condições mais higiênicas de manuseio. Além do uso continuo desses itens para assegurar o padrão de qualidade, também são desenvolvidos projetos e campanhas que incentivam o uso desses e outros equipamentos.

Inicialmente foram coletados os dados referentes ao consumo dos itens pertencentes a essa classe de materiais, durante o ano de 2017, e efetuou-se a classificação ABC.

A Tabela 1 apresenta a relação dos itens analisados com sua respectiva classificação e a Figura 3 traz sua representação gráfica.

Tabela 1. Dados utilizados ao para realizar a classificação $\mathrm{ABC}$ dos itens em estudo.

\begin{tabular}{|c|c|c|c|c|c|}
\hline Descrição do material & & $\begin{array}{l}\text { onsumo } \\
\text { alorizado }\end{array}$ & $\begin{array}{l}\text { Consumo } \\
\text { valorizado } \\
\text { acumulado }\end{array}$ & $\begin{array}{l}\% \text { Acumulada } \\
\text { do consumo }\end{array}$ & Classificação \\
\hline 1- Capa para Monobloco & $\mathrm{R} \$$ & $454.228,50$ & $\mathrm{R} \$ \quad 454.228,50$ & $39,74 \%$ & $\mathrm{~A}$ \\
\hline 2- Capa Descartavel & $\mathrm{R} \$$ & $202.089,56$ & $\begin{array}{ll}\mathrm{R} \$ & 656.318,06\end{array}$ & $57,42 \%$ & $\mathrm{~A}$ \\
\hline 3- Capa para Carrinho & $\mathrm{R} \$$ & $183.398,55$ & $\begin{array}{ll}\mathrm{R} \$ & 839.716,61 \\
\end{array}$ & $73,46 \%$ & $\mathrm{~A}$ \\
\hline 4- Capa para Cuba & $\mathrm{R} \$$ & $54.100,80$ & $\begin{array}{ll}\mathrm{R} \$ & 893.817,41 \\
\end{array}$ & $78,19 \%$ & $\mathrm{~A}$ \\
\hline 5- Luva G - Algodao & $\mathrm{R} \$$ & $49.744,80$ & $\begin{array}{ll}\mathrm{R} \$ & 943.562,21 \\
\end{array}$ & $82,55 \%$ & $\mathrm{~B}$ \\
\hline 6- Rolo Adesivo & $\mathrm{R} \$$ & $36.300,00$ & $\begin{array}{ll}\mathrm{R} \$ & 979.862,21\end{array}$ & $85,72 \%$ & $\mathrm{~B}$ \\
\hline 7-Touca Branca & $\mathrm{R} \$$ & $30.105,00$ & R\$ $1.009 .967,21$ & $88,36 \%$ & $\mathrm{~B}$ \\
\hline 8- Luva - Punho Longo & $\mathrm{R} \$$ & $28.480,00$ & $\mathrm{R} \$ 1.038 .447,21$ & $90,85 \%$ & $\mathrm{~B}$ \\
\hline 9- Luva G - Vinilica & $\mathrm{R} \$$ & $22.939,32$ & R\$ $1.061 .386,53$ & $92,85 \%$ & $\mathrm{~B}$ \\
\hline 10- Luva M - Vinilica & $\mathrm{R} \$$ & $20.049,48$ & $\mathrm{R} \$ 1.081 .436,01$ & $94,61 \%$ & $\mathrm{~B}$ \\
\hline 11- Mangotes - Branca & $\mathrm{R} \$$ & $16.944,00$ & R\$ 1.098.380,01 & $96,09 \%$ & $\mathrm{C}$ \\
\hline 12- Luva Gg - Vinilica & $\mathrm{R} \$$ & $15.786,00$ & $\mathrm{R} \$ 1.114 .166,01$ & $97,47 \%$ & $\mathrm{C}$ \\
\hline 13-Toucas Vermelhas & $\mathrm{R} \$$ & $15.070,00$ & $\mathrm{R} \$ 1.129 .236,01$ & $98,79 \%$ & $\mathrm{C}$ \\
\hline 14- Touca Azul & $\mathrm{R} \$$ & $3.852,00$ & $\mathrm{R} \$ 1.133 .088,01$ & $99,13 \%$ & $\mathrm{C}$ \\
\hline 15-Touca Verde & $\mathrm{R} \$$ & $3.480,00$ & $\mathrm{R} \$ 1.136 .568,01$ & $99,43 \%$ & $\mathrm{C}$ \\
\hline 16- Avental Manga Longa & $\mathrm{R} \$$ & $2.680,00$ & $\mathrm{R} \$ 1.139 .248,01$ & $99,67 \%$ & $\mathrm{C}$ \\
\hline 17-Touca Balaclava & $\mathrm{R} \$$ & $2.040,00$ & $\mathrm{R} \$ 1.141 .288,01$ & $99,84 \%$ & $\mathrm{C}$ \\
\hline 18- Sapatilha & $\mathrm{R} \$$ & $1.620,00$ & $\mathrm{R} \$ 1.142 .908,01$ & $99,99 \%$ & $\mathrm{C}$ \\
\hline 19- Mascara Bigodeira & $\mathrm{R} \$$ & 156,00 & $\mathrm{R} \$ 1.143 .064,01$ & $100,00 \%$ & C \\
\hline Total: & \multicolumn{2}{|c|}{$\mathrm{R} \$ 1.143 .064,01$} & & & \\
\hline
\end{tabular}

Anais do V CIMA I ech - 16 a 18 de outubro de 2018, rA IEC-SJC, Sao Jose dos Campos - SP. 
Figura 3. Representação gráfica do consumo valorizado acumulado dos itens em estudo

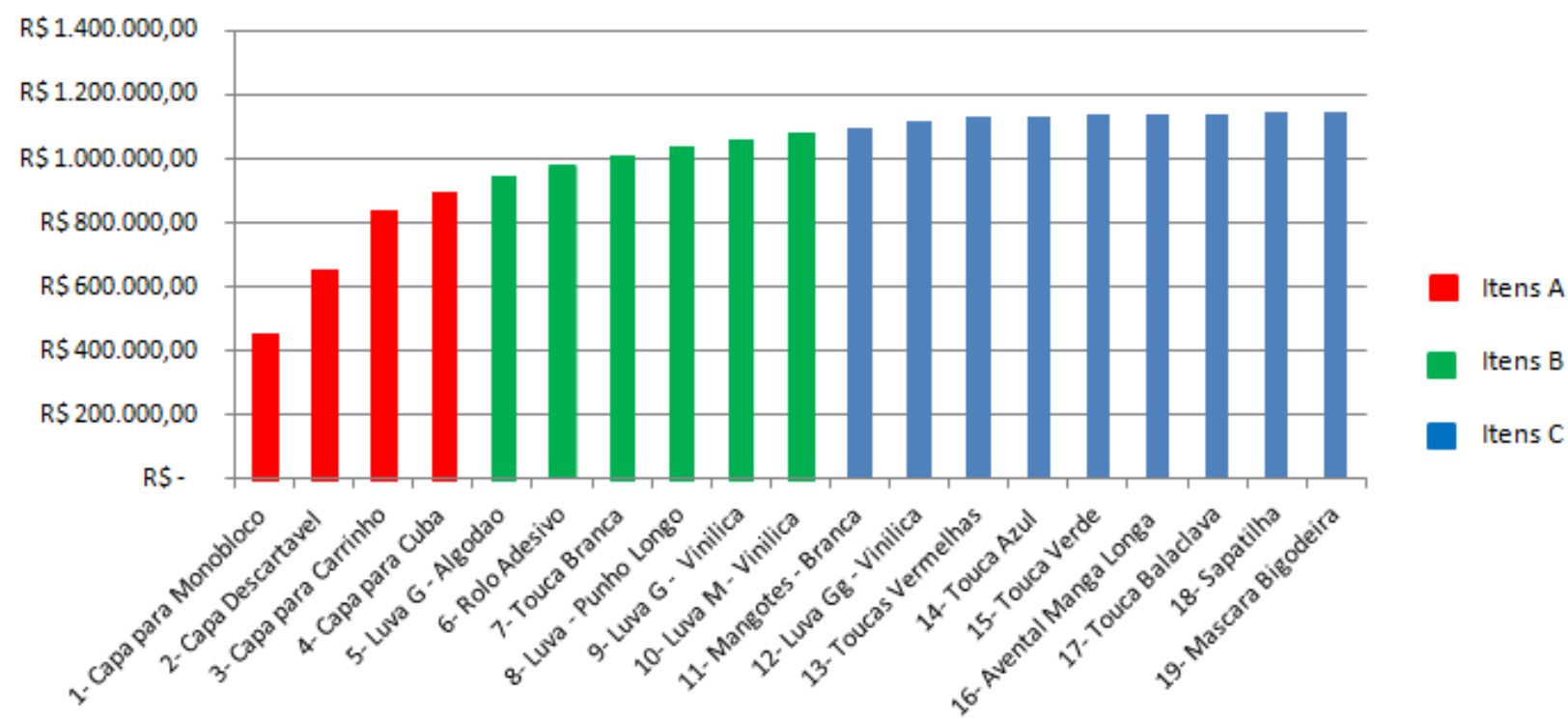

Para esse estudo, o foco será nos itens que garantem a qualidade dos produtos durante o processo de fabricação, mais especificamente no grupo "Toucas".

Existem quatro tipos de toucas que são de cores diferentes e utilizadas para identificar pessoas que transitam dentro da fábrica. São elas, as toucas brancas que identificam os funcionários, as toucas azuis sinalizam os visitantes, as toucas vermelhas são direcionadas à equipe de brigada de incêndio e por último, as toucas verdes, que são utilizadas por funcionários terceirizados.

Para desenvolver as análises relativas à gestão de estoque, foi selecionada a touca branca, utilizada para proteção dos produtos contra a queda de cabelo dos operadores durante o manuseio dos alimentos

Apesar de estar classificado como B, este item é considerado na organização estudada como sendo essencial e de grande importância, uma vez que sua falta impede a entrada de operadores na linha de produção por questões de garantia da qualidade dos produtos.

Utilizando os dados obtidos nas pesquisas realizadas na empresa, foi possível analisar as movimentações desse material do almoxarifado, conforme apresentado na Tabela 2

Tabela 2. Movimentações do item de estudo no ano de 2017.

\begin{tabular}{|c|c|c|}
\hline Mês & Entrada & Saída \\
\hline 01 & 56000 & 38200 \\
\hline 02 & 34000 & 54200 \\
\hline 03 & 30500 & 12500 \\
\hline 04 & & 9000 \\
\hline 05 & 15000 & 83000 \\
\hline 06 & 97000 & 23000 \\
\hline 07 & & 9000 \\
\hline 08 & 30000 & 12000 \\
\hline 09 & & 33000 \\
\hline 10 & 33000 & 18000 \\
\hline 11 & 30000 & 18000 \\
\hline 12 & & 9000 \\
\hline
\end{tabular}


Com base nas movimentações de saída do almoxarifado, apresentados na Tabela 2 acima, calculou-se a média, representada pela equação 3 e o desvio padrão representado pela equação 4 abaixo:

$$
\text { Média }=\frac{\Sigma \mathrm{X}}{\mathrm{N}}
$$

Onde :

$\Sigma \mathrm{x}=$ Somatória de todos elementos da amostra

$\mathrm{N}=$ Tamanho da amostra

$$
\text { Desvio padrão }=\sqrt{\frac{\sum(X i-m e ́ d i a)^{2}}{(n-1)}}
$$

Os valores obtidos foram 26.575 unidades para a média e $22.604,03$ unidades para o desvio padrão, conforme figura 4 .

Figura 4. Valores obtidos com a partir da somatória do consumo no ano de 2017.

\begin{tabular}{|c|c|c|}
\hline Somatória $(\Sigma \mathrm{X})$ & Média & Desvio \\
\hline 318900 & 26575 & 22604,03 \\
\hline
\end{tabular}

A Figura 5 representa as saídas do material do estoque ao longo de 2017 e a respectiva média calculada.

Figura 5. Representação gráfica da movimentação do item no ano de 2017.

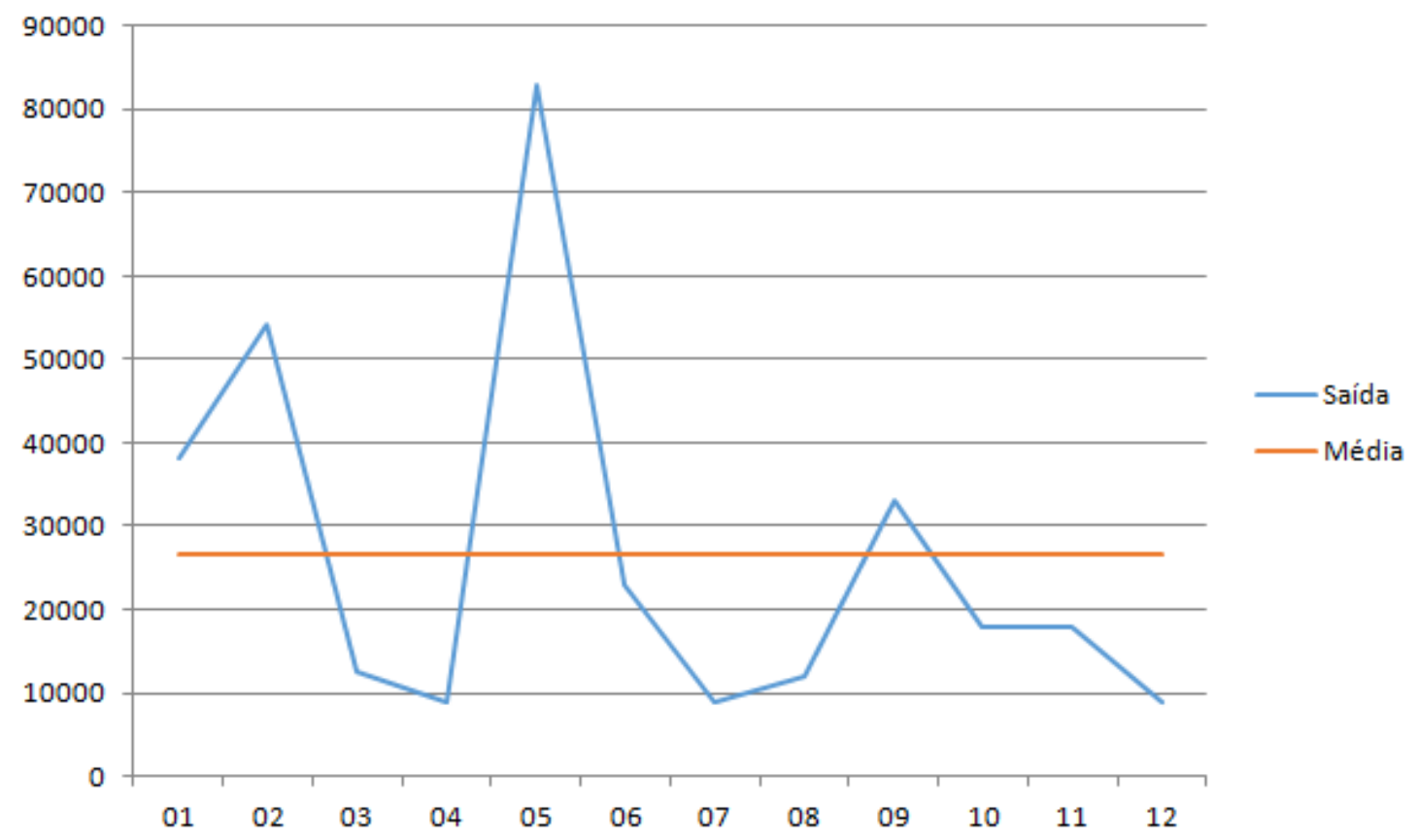

Anais do V CIMATech - 16 a 18 de outubro de 2018, FATEC-SJC, São José dos Campos - SP. 
Para calcular o estoque de segurança, é necessário considerar o nível de serviço, para obter-se da tabela da distribuição normal o valor de $Z \alpha$, a ser aplicado na Equação 1.

Considerando a criticidade e essencialidade do item analisado, foi estabelecido $99 \%$ de nível de serviço, ou seja, é aceitável que em $1 \%$ das retiradas ocorra a falta desse produto.

Vale salientar que, em casos emergenciais, o mesmo poderá ser substituído por toucas de outras cores.

O cálculo foi realizado utilizando-se a Equação 1 aplicada no cenário em estudo, onde:

$\mathrm{Z} \alpha=2,326$ (valor tabelado para um nível de atendimento de 99\%)

$\mathrm{D} \sigma=22.604$ unidades (desvio padrão calculado acima)

$\mathrm{TA}=1$ mês (Lead time do fornecedor)

$$
\begin{aligned}
& \mathrm{ES}=\mathrm{Z} \alpha * \mathrm{D} \sigma * \sqrt{\mathrm{TA}} \\
& \mathrm{ES}=2,326 * 22.604 * \sqrt{1} \\
& \mathrm{ES}=52.577 \text { unidades }
\end{aligned}
$$

Esse valor representa a quantidade que é capaz de atender a demanda durante o tempo de ressuprimento, absorvendo possíveis variações de consumo.

Após calcular o estoque de segurança, é possível encontrar o ponto de pedido e verificar qual o momento ideal para realizar uma nova solicitação do item para o fornecedor. Utiliza-se a Equação 2 aplicada no cenário da empresa em estudo, onde:

$\mathrm{CM}=26.575$ unidades (Consumo médio calculado acima)

$\mathrm{TR}=1$ mês (Lead time do fornecedor)

$\mathrm{ES}=52.577$ unidades (Obtido na Equação anterior)

$$
\begin{aligned}
& \mathrm{PP}=(\mathrm{CM} * \mathrm{TR})+\mathrm{ES} \\
& \mathrm{PP}=(26.575 * 1)+52.577 \\
& \mathrm{PP}=79.152 \text { unidades }
\end{aligned}
$$

Esse valor representa o nível de estoque que indica que um novo pedido de compra deve ser colocado para o fornecedor para atender a demanda futura deste material.

\section{CONCLUSÃO:}

Após as pesquisas bibliográficas e de campo, foi possível notar a grande importância do controle de estoque efetivo. A classificação ABC é essencial para entender quais os materiais que apresentam maior relevância dentro do almoxarifado da empresa. Desse modo os gestores conseguem ter uma melhor visão dos itens quanto ao seu custo e giro.

Considerando o item em estudo, após a elaboração da Tabela 1, notou-se que, quando considerado seu valor agregado e o consumo durante um período, ele é classificado como 
Item B. Portanto, é imprescindível realizar a comparação da classificação do item com sua necessidade dentro da empresa. A decisão de utilizar esse material como base para o estudo foi tomada devido à grande movimentação do mesmo e da importância dele na empresa, por questões de qualidade e melhor identificação dos colaboradores efetivos e desses os que fazem parte da equipe de apoio à brigada de incêndio, funcionários terceirizados e visitantes.

Utilizando-se de tabelas, gráficos e cálculos, obteve-se um demonstrativo quantitativo dos valores reais coletados de ponto de pedido e estoque de segurança do item em estudo, assim como também a média e o desvio padrão.

Apesar de ter sido utilizado apenas um item para estudo, pode-se utilizar o mesmo princípio para aplicação em outros materiais. Existem muitas oportunidades de melhoria na gestão do estoque desse grupo de materiais, de modo que é de suma importância entender a necessidade e utilização de cada um dos produtos individualmente antes de ampliar para os demais itens, pois cada um tem uma particularidade a ser considerada.

Manter um nível de estoque adequado contribui para que esses itens estejam sempre disponíveis e assim garantindo a qualidade do produto de modo que o cliente final esteja satisfeito com o produto entregue e mantenha sua fidelidade com a empresa.

\section{REFERÊNCIAS}

BALlOU, R. H. Gerenciamento da Cadeia de Suprimentos/Logística Empresarial. 5 Ed. Porto Alegre. Bookman, 2006.

BERTAGLIA, P. R. Logística e Gerenciamento da Cadeia de Abastecimento. 2 ed. São Paulo. Editora Saraiva, 2009.

BOWERSOX, D. J; CLOSS, D. J. Logística empresarial: o processo de integração da cadeia de suprimento. 1. ed., São Paulo: Atlas, 2001.

CHOPRA, S.; MEINDL, P. Gerenciamento da cadeia de suprimentos. Estratégia, planejamento e operação. 1. ed. Rio de Janeiro: Prentice Hall Brasil, 2003.

FONSECA, João José Saraiva da. Metodologia da pesquisa científica. Ceará: Universidade Estadual do Ceará, 2002.

GIL, Antonio Carlos. Como elaborar projetos de pesquisa. 4. ed. SÃo Paulo: Atlas, 2002. $173 \mathrm{p}$.

KOBAYASHI, S. Renovação da logística: como definir estratégias de distribuição física global. São Paulo: Atlas, 2000.

MACHADO, H. V.; ESPINHA, P. G. Reflexões sobre as dimensões do fracasso e mortalidade de pequenas empresas. Revista Capital Científico - Eletrônica, v. 3, n. 1, p. 5164, 2005.

NOVAES, Antônio Galvão. Logística e Gerenciamento da Cadeia de Distribuição. 4. ed. Rio de Janeiro: Elsevier - Campus, 2015. 415 p. 
SLACK, N.; CHAMBERS, S.; JOHNSTON, R. Administração da produção. 3 ed. São Paulo: Atlas, 2009.

WANKE, Peter. Gestão de Estoque na Cadeia de Suprimento: Decisões e Modelos Quantitativos. 3. ed. SÃo Paulo: Atlas, 2011.384 p 